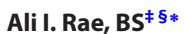

Amol Mehta, BA ${ }^{\text {? * }}$

Michael Cloney, MD, MPH"l

Connor J. Kinslow, BS"

Tony J.C. Wang, MD**

Govind Bhagat, MD ${ }^{\neq \neq}$

Peter D. Canoll, MD, PhD

George J. Zanazzi, MD, PhD

Michael B. Sisti, MD ${ }^{\S \S}$

Sameer A. Sheth, MD, PhD ${ }^{\S \S}$

E. Sander Connolly, MD $\$ \S$

Guy M. McKhann, MD ${ }^{\S \S}$

Jeffrey N. Bruce, MD ${ }^{\S \S}$

Fabio M. Iwamoto, MD

Adam M. Sonabend, MD\|

‡Warren Alpert Medical School, Brown University, Providence, Rhode Island; ${ }^{\S} \mathrm{De}$ partment of Health Policy, Mailman School of Public Health, Columbia University, New York, New York; "School of Medicine, University of Pittsburgh, Pittsburgh, Pennsylvania; Department of Neurological Surgery, Feinberg School of Medicine, Northwestern University, Chicago, Illinois; "College of Physicians and Surgeons, Columbia University, New York, New York; ** Department of Radiation Oncology, College of Physicians and Surgeons, Columbia University Medical Center, New York, New York \#‡Department of Pathology and Cell Biology, College of Physicians and Surgeons, Columbia University Medical Center, New York, New York; ${ }^{\S}$ Department of Neurological Surgery, College of Physicians and Surgeons, Columbia University Medical Center, New York New York; " Department of Neurology, College of Physicians and Surgeons, Columbia University Medical Center, New York, New York

*These authors contributed equally to this work.

A preliminary version of this study was accepted by the Congress of Neurological Surgeons (October 7-10, 2017, Boston, Massachusetts) and the Society of Neurooncology 2017 meeting (November 16-19, 2017, San Francisco,

California) as an abstract.

\section{Correspondence:}

Adam M. Sonabend, MD,

Department of Neurosurgery,

Northwestern University,

Feinberg School of Medicine,

676 N St. Clair Street, Suite 2210

Chicago IL 60611.

E-mail: adam.sonabend@nm.org

Received, August 13, 2017.

Accepted, February 28, 2018.

Published Online, April 4, 2018.

Copyright (C) 2018 by the

Congress of Neurological Surgeons

\title{
Craniotomy and Survival for Primary Central Nervous System Lymphoma
}

BACKGROUND: Cytoreductive surgery is considered controversial for primary central nervous system lymphoma (PCNSL).

OBJECTIVE: To investigate survival following craniotomy or biopsy for PCNSL

METHODS: The National Cancer Database-Participant User File (NCDB, $n=8936)$, Surveillance, Epidemiology, and End Results Program (SEER, $n=4636$ ), and an institutional series (IS, $n=132$ ) were used. We retrospectively investigated the relationship between craniotomy, prognostic factors, and survival for PCNSL using case-control design.

RESULTS: In NCDB, craniotomy was associated with increased median survival over biopsy (19.5 vs $11.0 \mathrm{mo}$ ), independent of subsequent radiation and chemotherapy (hazard ratio [HR] $0.80, P<.001)$. We found a similar trend with survival for craniotomy vs biopsy in the IS (HR $0.68, P=.15$ ). In SEER, gross total resection was associated with increased median survival over biopsy ( 29 vs $10 \mathrm{mo}, \mathrm{HR} 0.68, P<.001$ ). The survival benefit associated with craniotomy was greater within recursive partitioning analysis (RPA) class 1 group in NCDB (95.1 vs $29.1 \mathrm{mo}, \mathrm{HR} 0.66, P<.001$ ), but was smaller for RPA 2-3 (14.9 vs $10.0 \mathrm{mo}, \mathrm{HR} 0.86$, $P<.001)$. A surgical risk category $(\mathrm{RC})$ considering lesion location and number, age, and frailty was developed. Craniotomy was associated with increased survival vs biopsy for patients with low RC (133.4 vs $41.0 \mathrm{mo}, \mathrm{HR} 0.33, P=.01)$, but not high RC in the IS.

CONCLUSION: Craniotomy is associated with increased survival over biopsy for PCNSL in 3 retrospective datasets. Prospective studies are necessary to adequately evaluate this relationship. Such studies should evaluate patients most likely to benefit from cytoreductive surgery, ie, those with favorable RPA and RC.

KEY WORDS: CNS, Lymphoma, Resection, Survival, Prognosis

Neurosurgery 84:935-944, 2019

DOI:10.1093/neuros/nyy096

www.neurosurgery-online.com

P rimary central nervous system lymphoma (PCNSL) accounts for $1 \%$ to $2 \%$ of all primary central nervous system (CNS) tumors. ${ }^{1}$ PCNSL carries poor prognosis, with

ABBREVIATIONS: ACS, American College of Surgeons; $\mathrm{CoC}$, Commission on Cancer; $\mathrm{Cl}$, confidence interval; CNS, central nervous system; GTR, gross total resection; HR, hazard ratio; ICD 0-3, International Classification of Diseases for Oncology, third edition; IS, institutional series; KPS, Karnofsky Performance Score; NCDB, The National Cancer Database; OR, odds ratio; OS, overall survival; $\mathrm{PH}$, proportional hazard; $\mathbf{P C N S L}$, primary central nervous system lymphoma; $\mathbf{R C}$, risk category; $\mathbf{R P A}$, recursive partitioning analysis; SEER, Surveillance, Epidemiology, and End Results; STR, subtotal resection; TTR, total tumor resection

Supplemental digital content is available for this article at www.neurosurgery-online.com. 5-yr survival of $15 \%$ to $30 \% .^{2-4}$ The current management paradigm for patients with PCNSL involves stereotactic needle biopsy for diagnosis followed by systemic high-dose methotrexatebased chemotherapy. ${ }^{5}$ Surgery for cytoreduction is not standard for PCNSL, though it is occasionally performed for symptomatic relief of severe mass effect or if the lesion mimics other pathology on imaging studies. ${ }^{5,6}$ This treatment paradigm contrasts with the management of other intra-axial tumors including brain metastasis and diffusely infiltrative gliomas, where surgery contributes to oncologic control and is associated with a survival advantage. ${ }^{7-12}$

Cytoreductive surgery was excluded from first-line management of PCNSL largely due to results from studies concluding resection offered no benefit and potentially worsened outcomes. ${ }^{13-25}$ However, many of these studies 
had relatively small sample sizes and were conducted prior to the modern neurosurgical era. Currently, several technological advancements such as fluorescent tumor visualization, magnetic resonance imaging, neuronavigation, and intraoperative monitoring have contributed to improved outcomes for intra-axial neurosurgery. ${ }^{26-28}$ Additionally, many of the studies discouraging resection of PCNSL were conducted prior to the standardization of high-dose systemic methotrexate, ${ }^{13-17}$ a major component of the current treatment for PCNSL. Evaluation of the therapeutic benefit of cytoreductive surgery warrants further analysis in this context. ${ }^{6}$

We investigated the association between craniotomy and survival for PCNSL in contemporary series that complement each other in sample size and granularity of clinical variables. Our institutional series (IS) includes data from 132 patients and contains detailed information on clinical parameters. We crossvalidated these findings using data from 8936 patients in the National Cancer Database-Participant User File (NCDB) and 4636 patients in Surveillance, Epidemiology, and End Results Program (SEER).

\section{METHODS}

\section{Institutional Series}

After receiving patient consent and institutional review board approval, we collected data on patients diagnosed with PCNSL at our institution between 2000 and 2017. We excluded patients with lymphoma outside the CNS and those with spinal lymphoma. We retrospectively reviewed patient records for clinical information, including age at diagnosis, comorbidities, lesion characteristics, and survival.

\section{National Cancer Database-Participant User File}

The NCDB is a retrospective nationwide dataset sponsored by the American College of Surgeons (ACS) and the American Cancer Society, constituting $70 \%$ of incident invasive cancer cases in the United States. ${ }^{29}$ Data were collected at over 1500 Commission on Cancer (CoC)accredited hospitals between 2004 and 2013. This database has been validated for several variables. ${ }^{30}$ We identified patients diagnosed with non-Hodgkin's lymphoma (International Classification of Diseases for Oncology, third edition [ICD O-3] codes 9590-9599, 9670-9699, 97009719, 9720-9729, 9827) within the CNS (ICD O-3 codes C71.0C71.9) between 2004 and 2013. Patients lacking histological confirmation, having non-CNS primary site, unknown follow-up, or disease involving the meninges, spine, or optic nerves were excluded.

Age was evaluated as a continuous variable in whole years. CharlsonDeyo score was coded equivalently to Charlson comorbidity score, except Charlson-Deyo score of 2 was coded for Charlson score $\geq 2$. Radiation was coded as external beam therapy vs no radiation. Chemotherapy was coded as single or multi-agent chemotherapy vs no chemotherapy. NCDB lacks information on specific chemotherapeutic agents. The data used in the study are derived from a de-identified NCDB file. The ACS and the $\mathrm{CoC}$ have not verified and are not responsible for the analytic or statistical methodology employed, or the conclusions drawn from these data by the investigator.

\section{Surveillance, Epidemiology, and End Results Program}

SEER is a population-based tumor registry sponsored by the National Cancer Institute, covering approximately $28 \%$ of the United States population. ${ }^{30}$ We identified patients diagnosed with non-Hodgkin's lymphoma (ICD O-3 codes 9590-9599, 9670-9699, 9700-9719, 97209729, 9827) within the CNS (ICD O-3 codes C70.1-C72.9) between 1995 and 2013. We excluded patients lacking histological confirmation and those diagnosed at autopsy.

\section{Recursive Partitioning Analysis Class}

We stratified patients by Memorial Sloan Kettering recursive partitioning analysis (RPA) classes ${ }^{31}$ which are delineated as follows: class 1 (patients $<50 \mathrm{yr}$ old); class 2 (patients $\geq 50 \mathrm{yr}$ old with Karnofsky Performance Score $[\mathrm{KPS}] \geq 70$ ); and class 3 (patients $\geq 50 \mathrm{yr}$ old with KPS < 70).

\section{Risk Category Classification}

Risk category (RC) is a scale we designed to incorporate age, frailty, single vs multiple lesions, and superficial vs deep brain lesion location. Number of lesions and lesion location have been previously shown to be independently predictive of surgical complications. ${ }^{32}$ Lesions involving brainstem, basal ganglia, corpus callosum, or periventricular areas were classified as deep. Departing from the prognostic system of Ferreri et $\mathrm{al}^{33}$ we classified the cerebellum as superficial despite worse prognosis because of its relative surgical accessibility. Frailty has been validated in geriatric patients to approximate health status ${ }^{34,35}$ and is defined as physiologic vulnerability to adverse events. ${ }^{34}$ Frailty affects complication rates after intracranial surgery. ${ }^{32,36}$ The modified frailty index was developed from the Canadian Study on Health and Aging 37,38 with increasing frailty score associated with poorer outcomes across many surgical procedures, ${ }^{39-41}$ including craniotomy for glioblastoma resection. ${ }^{36}$ This index gives 1 point for each variable present: difficulty with activities of daily living; history of diabetes mellitus; lung or respiratory disease; congestive heart failure; myocardial infarction; other cardiac disease; arterial hypertension; clouding, delirium, or cognitive impairment; history of transient ischemic attack; history of stroke; and peripheral vascular disease. To account for operative selection factors, RC adds 1 point each for age $>55 \mathrm{yr}$, multiple lesions, and deep lesion location (Figure, Supplemental Digital Content 1). We grouped patients into low-RC (0-3 points) and high-RC ( $\geq 4$ points) groups.

\section{Statistical Analysis}

Analysis was performed using STATA version 14 (StataCorp LLC, College Station, Texas). Kaplan-Meier plots were generated from STATA software. Descriptive statistics, t-tests, Fisher's exact tests, chi-squared tests, log-rank tests, and Cox proportional hazards $(\mathrm{PH})$ analysis (Breslow method for ties) were used as appropriate and are reported in tables. Missing data were treated by omitting specific data points without removing observations. Multivariable Cox regression was performed in stepwise fashion after fitting regressions for each variable, with $P<.05$ as entry criterion and $P>.20$ as removal criterion. Data were rightcensored. Vital status and time to death or censorship were coded using NCDB variables. Tests with 2 -tailed; $P<.05$ were considered statistically significant.

$\mathrm{PH}$ assumption test returned $P<.01$, indicating non- $\mathrm{PH}$. $\mathrm{PH}$ assumption violations do not invalidate the model if there is clinical or biological explanation for varied hazard over time, and the hazard ratio remains a good effect measure. ${ }^{42}$ As expected, our study shows 
highest incidence of PCNSL in older patients. We expect PH assumption violation due to increase in deaths from causes other than PCNSL late in follow-up period. We performed $\mathrm{PH}$ assumption test for each variable, then plotted these on log-log plots and Kaplan-Meier vs predicted survival plots (Figure, Supplemental Digital Content 2). Landmark analyses at 30 and $90 \mathrm{~d}$ for each variable with shorter follow-up time returned valid $\mathrm{PH}$ assumption. $\mathrm{PH}$ assumption violation only occurred late in follow-up period, consistent with clinical prediction.

\section{RESULTS}

\section{Baseline Patient Characteristics}

The IS included 132 patients. Multiple lesions (odds ratio [OR] $0.38, P=.009$ ) and deep brain lesion location (OR 0.27, $P<.001)$ were associated with higher odds of biopsy than craniotomy.

In NCDB, we identified 8936 patients matching study criteria. Patients were slightly more likely to undergo biopsy over craniotomy if they had deep lesions (OR 0.60, $P=.004$ ) or higher Charlson-Deyo score (OR 0.85, $P=.009$ ).

In SEER, 4636 patients matching study criteria were included. The biopsy group had a higher proportion of males than the gross total resection (GTR) group $(58.5 \%$ vs $51.3 \%, P<.001)$ and subtotal resection (STR) group $(58.5 \%$ vs $52.8 \%, P<.001)$.

Stratified baseline patient characteristics for each dataset are summarized in Table 1.

\section{Relationship Between Craniotomy vs Biopsy and Survival}

In NCDB, patients who underwent craniotomy had longer median survival $(19.5 \mathrm{mo}, 95 \%$ confidence interval [CI; 16.8, $22.0])$ vs biopsy (11.0 mo, 95\% CI [10.1, 12.3]; hazard ratio [HR] 0.83, 95\% CI [0.79, 0.88], $P<.001$; Figure 1A). In multivariable analysis, craniotomy (HR 0.80, 95\% CI [0.75, 0.84], $P<.001$ ), age (HR 1.03 for each 1 -yr increase, 95\% CI [1.03, 1.03], $P<.001$ ), lower Charlson-Deyo score (HR 1.18, 95\% CI $[1.14,1.25], P<.001$ ), receiving chemotherapy (HR 0.40 , $95 \% \mathrm{CI}[0.37,0.42], P<.001)$ and receiving radiation therapy [HR 0.90, 95\% CI [0.84, 0.95], $P<.001)$ were independently predictive of survival. Deep vs superficial lesion location was not predictive of survival in univariable or multivariable analysis (Table 2). In IS, median survival was 46.0 mo (95\% CI [35.7, 133.4]) with craniotomy vs 24.7 mo (95\% CI [13.8, 54.9]) with biopsy (HR 0.68, 95\% CI [0.39, 1.16], $P=.15$; Figure $1 \mathrm{~B}$ ).

We analyzed SEER to investigate whether extent of resection influenced the association between craniotomy and survival. Median survival was 29 mo for GTR (95\% CI [24, 34]), 24 mo for STR $(95 \%$ CI $[13,40])$, and 10 mo for biopsy $(95 \%$ CI [10, 12]; Figure 1C). Resection was associated with survival benefit over biopsy for both GTR (HR 0.68, 95\% CI [0.62, 0.74], $P<.001]$ and STR [HR 0.73, 95\% CI [0.61, 0.89], $P=.001)$ groups.

\section{Combined Effect of Craniotomy and Chemotherapy on Survival}

We found that combining craniotomy and chemotherapy was associated with an additive increase in survival. Median survival was 25.1 mo with chemotherapy and biopsy, and 37.4 mo with chemotherapy and craniotomy (log-rank $P<.001$; Figure 2). This effect remained when the model was adjusted for age.

\section{Effect of Recursive Partitioning Analysis on the Relationship Between Craniotomy and Survival}

Because NCDB lacks information on KPS, we could only stratify patients into RPA class 1 or RPA 2-3 in NCDB, but these results were complemented by analysis of all classes in IS. Median survival was $46.9 \mathrm{mo}(95 \% \mathrm{CI}[36.7,57.6])$ in the RPA 1 group vs 11.4 mo $(95 \%$ CI $[10.5,12.5])$ in the RPA 2-3 group (HR 0.58, 95\% CI [0.54, 0.63], $P<.001$; Figure, Supplemental Digital Content 3A). In IS, median survival was not reached at 135 mo of follow-up for RPA class 1. Median survival was 37.2 mo for RPA class 2 and 16.5 mo for RPA class 3 (HR 0.52 for each class decrease, $95 \%$ CI $[0.35,0.79], P=.002$; Figure, Supplemental Digital Content 3B). These results replicate findings by Abrey et $\mathrm{al}^{31}$ and validate RPA as a prognostic indicator for PCNSL.

We investigated whether RPA class influenced survival differences between craniotomy and biopsy. Within the RPA1 group in NCDB, craniotomy was associated with median survival of 95.1 mo $(95 \%$ CI $[61.2,112.7])$ vs 29.1 mo for biopsy (95\% CI [17.6, 37.6]; HR 0.66, 95\% CI [0.57, 0.77], $P<.001$; Figure 3A). The IS showed a similar trend (HR 0.17, 95\% CI [0.02, 1.45], $P=.10$; Figure $3 \mathrm{~B})$. We next evaluated the effect of worse prognosis (clustering RPA class 2-3) on survival by surgery type. There was a smaller but significant survival benefit associated with craniotomy for RPA 2-3 patients in NCDB, with median survival of 14.9 mo $(95 \%$ CI $[12.9,16.8])$ for craniotomy, and $10.0 \mathrm{mo}$ $(95 \%$ CI $[9.0,10.9])$ for biopsy (HR $0.86,95 \%$ CI $[0.81,0.91]$, $P<.001$; Figure 3C). In IS, RPA 2-3 showed no difference in survival for craniotomy vs biopsy $(P=.53$; Figure $3 \mathrm{D})$.

\section{Effect of RC on the Relationship Between Craniotomy and Survival}

Because RPA classification does not account for patient and lesion characteristics that might influence whether craniotomy or biopsy is performed, we designed an RC that incorporates these factors. We then evaluated the relationship between craniotomy and survival in patients with similar RC. The low-RC group had median survival of 76.0 mo $(95 \%$ CI $[41.0,133.4])$ vs 19.3 mo $(95 \%$ CI $[11.0,29.3])$ for high-RC (HR $0.43,95 \%$ CI $[0.25$, $0.75], P=.003$; Figure 4A). Low- $\mathrm{RC}$ was associated with survival independent of RPA class on multivariable analysis of IS (HR $0.52,95 \%$ CI $[0.28,0.93], P=.03$ ).

We compared survival after craniotomy or biopsy in low-RC patients in IS. Median survival was 133.4 mo (95\% CI [46.0, 
TABLE 1. Baseline Patient Characteristics of Included Observations From the NCDB, IS, and SEER Databases

\begin{tabular}{|c|c|c|c|c|c|c|c|c|c|c|}
\hline & \multicolumn{3}{|c|}{ IS } & \multicolumn{3}{|c|}{ NCDB } & \multicolumn{4}{|c|}{ SEER } \\
\hline & $\begin{array}{c}\text { Biopsy } \\
(n=72)\end{array}$ & $\begin{array}{c}\text { Craniotomy } \\
(\mathrm{n}=60)\end{array}$ & $P$-value & $\begin{array}{c}\text { Biopsy } \\
(n=5513)\end{array}$ & $\begin{array}{l}\text { Craniotomy } \\
(\mathrm{n}=3423)\end{array}$ & $P$-value & $\begin{array}{c}\text { Biopsy } \\
(n=3350)\end{array}$ & $\begin{array}{c}\text { STR } \\
(\mathrm{n}=216)\end{array}$ & $\begin{array}{c}\text { GTR } \\
(n=1070)\end{array}$ & $P$-value \\
\hline Median survival (95\% Cl) & \multicolumn{3}{|c|}{$37.2 \mathrm{mo}(21.9,76.0)$} & \multicolumn{3}{|c|}{$13.5 \mathrm{mo}(12.6,14.5)$} & \multicolumn{4}{|c|}{$15 \mathrm{mo}(14,17)$} \\
\hline Median age & 67 & 63 & & 65 & 65 & & 62 & 65 & 63 & \\
\hline Number male & $35(48 \%)$ & $27(45 \%)$ & $P=.68$ & $\begin{array}{l}2859 \\
(51.9 \%)\end{array}$ & $\begin{array}{l}1703 \\
(49.8 \%)\end{array}$ & $P=.06$ & $\begin{array}{l}1959 \\
(58.5 \%)\end{array}$ & $114(52.8 \%)$ & $549(51.3 \%)$ & $P<.001^{*}$ \\
\hline \multicolumn{11}{|l|}{ Histology } \\
\hline B-cell & $61(85 \%)$ & $53(88 \%)$ & $P=.73$ & $\begin{array}{l}4917 \\
(89.2 \%)\end{array}$ & $\begin{array}{l}3088 \\
(90.2 \%)\end{array}$ & $P=.12$ & $\begin{array}{l}2797 \\
(83.5 \%)\end{array}$ & $186(86.1 \%)$ & 930 (86.9\%) & $P=.02$ \\
\hline Other & $11(61 \%)$ & $7(12 \%)$ & & $596(10.8 \%)$ & 335 (9.8\%) & & $553(16.5 \%)$ & $30(13.9 \%)$ & $140(13.1 \%)$ & \\
\hline \multicolumn{11}{|l|}{ Radiation therapy } \\
\hline External beam radiation & $8(80 \%)$ & $8(67 \%)$ & $P=.83$ & $\begin{array}{l}1548 \\
(28.3 \%)\end{array}$ & $946(28.2 \%)$ & $P=.92$ & & & & \\
\hline No radiation & $2(20 \%)$ & $4(33 \%)$ & & 3919 (71.7\%) & $\begin{array}{l}2406 \\
(71.8 \%)\end{array}$ & & & & & \\
\hline \multicolumn{11}{|l|}{ Chemotherapy } \\
\hline Received chemo & $25(89 \%)$ & $23(92 \%)$ & $P=.74$ & $\begin{array}{l}3605 \\
(73.4 \%)\end{array}$ & $\begin{array}{l}2094 \\
(71.3 \%)\end{array}$ & $P=.05$ & & & & \\
\hline No chemo & $3(11 \%)$ & $2(8 \%)$ & & $\begin{array}{l}1308 \\
(26.6 \%)\end{array}$ & $844(28.7 \%)$ & & & & & \\
\hline \multicolumn{11}{|l|}{ Location } \\
\hline Deep & 51 (71\%) & $24(40 \%)$ & $P<.001$ & $93(4.5 \%)$ & $53(2.8 \%)$ & $P=.004$ & & & & \\
\hline Superficial & $21(29 \%)$ & $36(60 \%)$ & & $\begin{array}{l}1976 \\
(95.5 \%)\end{array}$ & $\begin{array}{l}1879 \\
(97.2 \%)\end{array}$ & & & & & \\
\hline \multicolumn{11}{|l|}{ RPA class } \\
\hline 1 & $14(20 \%)$ & $12(20 \%)$ & & $972(17.6 \%)$ & $576(16.8 \%)$ & & & & & \\
\hline $2(\geq 2$ in NCDB) & $33(45 \%)$ & $35(58 \%)$ & $P=.23$ & $\begin{array}{l}4541 \\
(82.4 \%)\end{array}$ & $\begin{array}{l}2874 \\
(83.2 \%)\end{array}$ & $P=.25$ & & & & \\
\hline 3 & $25(35 \%)$ & $13(22 \%)$ & & N/A & $\mathrm{N} / \mathrm{A}$ & & & & & \\
\hline Comorbid diagnosis & 0.3 & 0.14 & $P=.19$ & & & & & & & \\
\hline \multicolumn{11}{|l|}{ Charlson-Deyo score } \\
\hline 0 & & & & $\begin{array}{l}3486 \\
(63.2 \%)\end{array}$ & $\begin{array}{l}2460 \\
(65.3 \%)\end{array}$ & & & & & \\
\hline 1 & & & & $\begin{array}{l}1146 \\
(20.8 \%)\end{array}$ & $687(18.8 \%)$ & $P=.003$ & & & & \\
\hline 2 & & & & $881(16.0 \%)$ & 571 (15.9\%) & & & & & \\
\hline \multicolumn{11}{|l|}{ Number of lesions } \\
\hline Single & $34(47 \%)$ & $42(80 \%)$ & $P=.009$ & & & & & & & \\
\hline Multiple & $38(52 \%)$ & $18(30 \%)$ & & & & & & & & \\
\hline \multicolumn{11}{|l|}{ Maximum dimension } \\
\hline$\geq 3 \mathrm{~cm}$ & $23(32 \%)$ & $21(35 \%)$ & $P=1$ & & & & & & & \\
\hline$<3 \mathrm{~cm}$ & $27(38 \%)$ & $24(40 \%)$ & & & & & & & & \\
\hline \multicolumn{11}{|l|}{ Risk category } \\
\hline Low RC (0-3) & $33(46 \%)$ & $39(65 \%)$ & $P=.03$ & & & & & & & \\
\hline High RC (4+) & 39 (54\%) & $21(35 \%)$ & & & & & & & & \\
\hline
\end{tabular}

Italic font indicates that $\chi^{2}$ statistic was significant. Higher Charlson-Deyo score indicates higher comorbidity: $0=$ Charlson score of $0,1=$ Charlson score of $1,2=$ Charlson score $\geq 2$. $\mathrm{GTR}=$ gross total resection, STR = subtotal resection. Location was coded as "superficial" for lesions confined to cerebellum or frontal, parietal, temporal, or occipital lobes. Brainstem and periventricular or intraventricular lesions were classified as "deep." We have reported count and proportion for categorical variables as well as mean and standard deviation for continuous variables. *Pairwise comparisons were done between GTR vs biopsy and STR vs biopsy, and both returned $P<.001$. 
133.4]) with craniotomy vs 41.0 mo (95\% CI $[6.3,100.0])$ with biopsy (HR 0.33, 95\% CI [0.14, 0.80], $P=.01$; Figure 4B). In contrast, there was a trend toward shorter survival in high-RC patients who underwent craniotomy vs biopsy (HR 1.90, 95\% CI $[0.93,3.88], P=.08$; Figure 4C).

\section{Surgeon Intent of Cytoreduction}

After reviewing operative reports of all craniotomy cases in IS, we identified surgeon's intention to perform cytoreduction in 51 of 60 cases. The most common rationale for resection was diagnostic uncertainty, followed by the ability to safely resect obvious tumor in order to mitigate mass effect or the likelihood of future mass effect. Fourteen of the 51 cases in which the surgeon intended to perform cytoreduction were classified as GTR. The remaining 37 STR were performed with the goal of maximal safe resection, with surgical or anatomical considerations cited as rationale for not obtaining GTR.

For the 9 cases in which the surgeon performed craniotomy but seemingly did not intend to perform cytoreduction, resection was discontinued due to intraoperative frozen section consistent with PCNSL after multiple biopsies or minor excisions.

\section{DISCUSSION}

Older studies established the current treatment paradigm discouraging surgery for PCNSL. ${ }^{6,13-25}$ However, recent studies have highlighted the potential role for cytoreductive surgery for this disease. ${ }^{43,44}$ Using complementary institutional and population-based analyses, we found that craniotomy is associated with survival benefit over biopsy in patients with PCNSL. The survival benefit associated with craniotomy remains independent of chemotherapy, radiotherapy, and baseline prognostic factors. In NCDB, patients who underwent craniotomy had almost doubling of median survival time over patients who had biopsy only. In SEER, both GTR and STR were associated with increased survival over biopsy, and there was a trend toward longer survival with more extensive resection.

Patients with better prognostic factors had an even longer survival benefit with craniotomy. In NCDB, RPA class 1 patients undergoing craniotomy had over 3-fold increase in median survival time, with a similar trend in IS. To incorporate surgical considerations, we created an RC that was predictive of survival and found that craniotomy in low-RC patients more than tripled the median survival time compared to biopsy. This novel clinical scale is illustrated in Table 3 .

Weller et $\mathrm{al}^{43}$ first demonstrated an association between craniotomy and survival over biopsy for PCNSL, yet as a post hoc analysis of a clinical trial, this study was subject to selection bias and lacks generalizability. Jelicic et $\mathrm{al}^{44}$ demonstrated that total tumor resection (TTR) was significantly associated with increased overall survival (OS), but they lacked the follow-up to reach median OS in the TTR group. Our study validates these findings and demonstrates a more robust effect in a much larger population. Additionally, we identified that patients with better

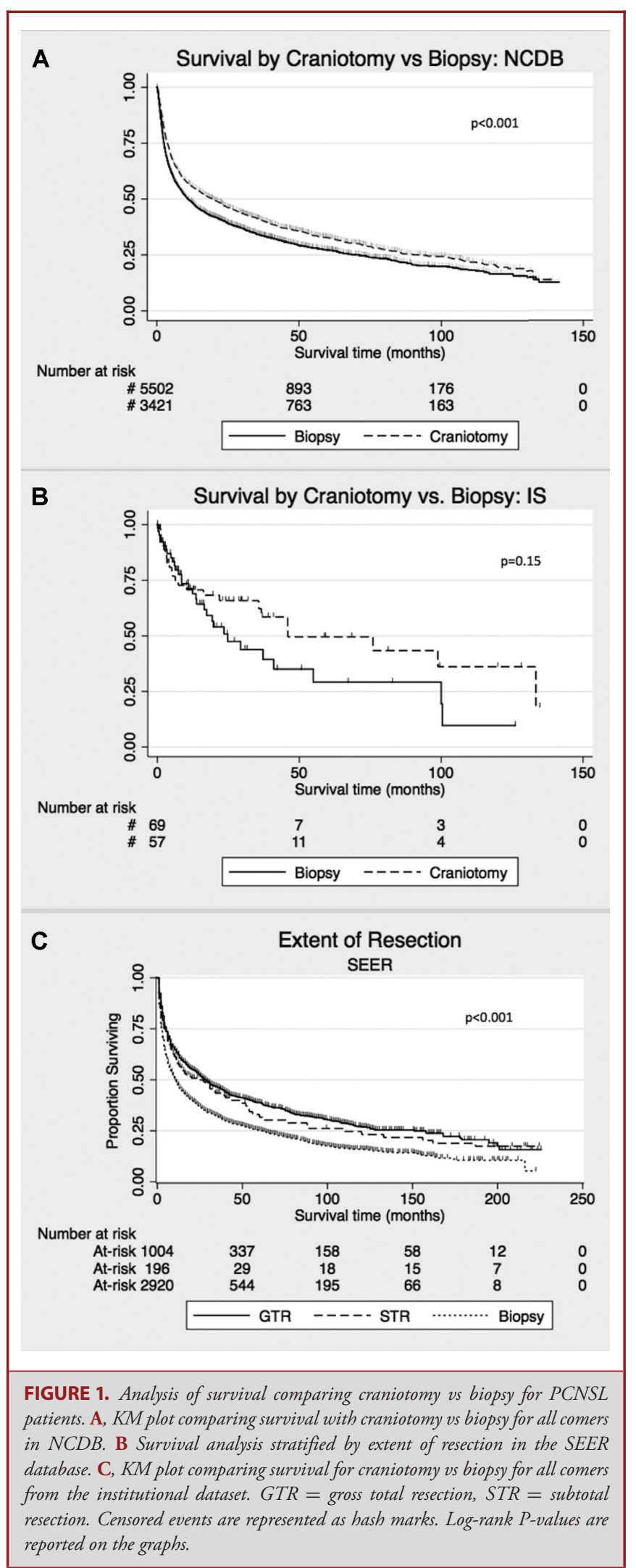


TABLE 2. Multivariable Analysis in the NCDB

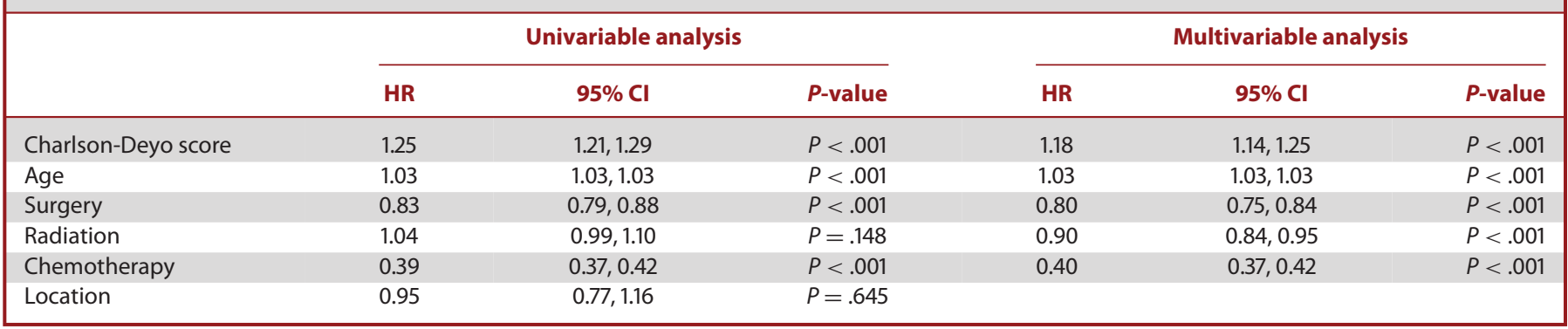

Charlson-Deyo score is equivalent to Charlson comorbidity score, except Charlson-Deyo score of 2 indicates Charlson score $\geq 2$. Age was evaluated as a continuous variable in whole years. Surgery is coded as craniotomy vs biopsy. Radiation was coded as external beam therapy vs no radiation and chemotherapy was coded as single or multi-agent chemotherapy vs no chemotherapy based on available data. Location was coded as superficial or deep.

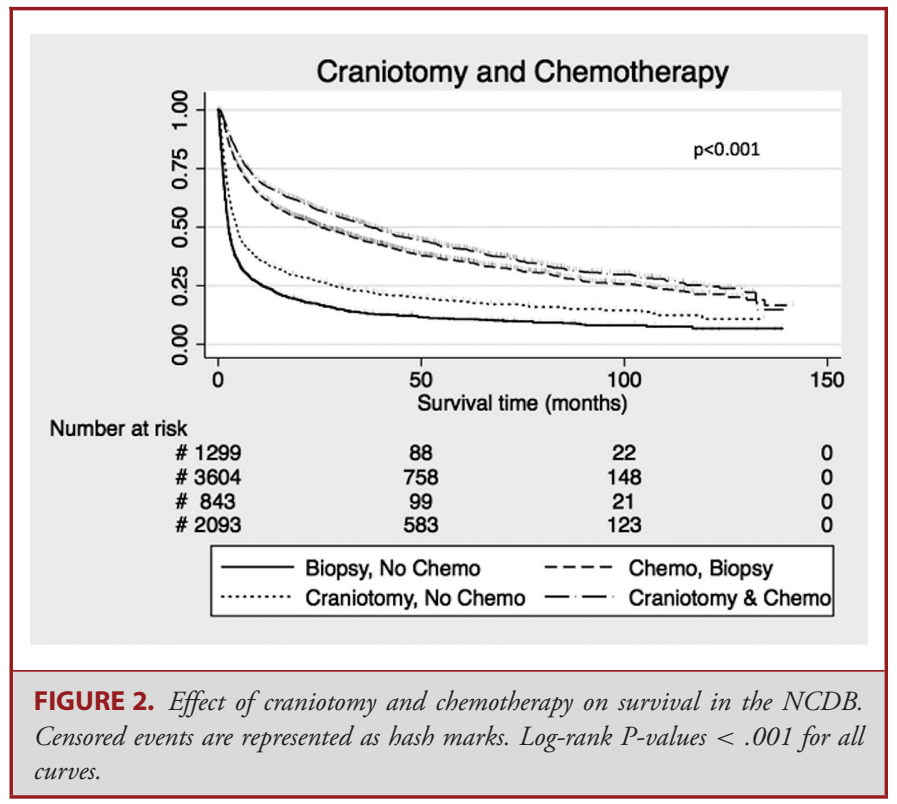

RPA and low-RC were associated with longer survival in the setting of craniotomy.

While most lymphomas are treated with systemic therapy, evidence suggests that cytoreductive surgery may play a role in treatment for non-CNS lymphoma. Resection in addition to chemotherapy increases survival over chemotherapy alone for patients with intestinal diffuse large B-cell lymphoma. ${ }^{45}$ In a recent retrospective cohort study, 2-yr OS for patients undergoing complete resection of intra-abdominal follicular lymphoma was similar to those treated with chemotherapy. ${ }^{46}$ For PCNSL, available chemotherapeutic regimens are not as effective as those for systemic lymphoma. Additionally, CNS tumors can cause high morbidity and mortality due to their location. Resection can rapidly improve neurological symptoms and increase the window of time for medical therapy to become effective.
Consequently, multimodality treatment is likely beneficial for patients with this disease, as suggested by our finding of significant median survival increase with both craniotomy and chemotherapy vs chemotherapy alone.

The retrospective nature of this study may introduce welldescribed biases in data collection and analysis. We could not control for some variables that may affect survival in this disease such as tumor molecular profiles. ${ }^{47,48}$ Resectability is another consideration; more resectable lesions may confer better prognosis, irrespective of surgery type. We attempted to address this possible confounder using RC stratification; however, we recognize that resectability is a complex variable that is poorly represented by numerical scales. ${ }^{49}$

Factors influencing patient selection for craniotomy or biopsy may also affect survival. Large-scale datasets lack the information to evaluate these parameters. Although many patients in IS who underwent biopsy also had worse prognostic factors, our RC analysis addresses some of these limitations by grouping patients with similar preoperative and lesion characteristics.

\section{Limitations}

The nation-wide datasets we analyzed have been advocated for use in the clinical evaluation of rare diseases like PCNSL. ${ }^{29}$ While this approach has many advantages, inherent limitations include missing data, the possibility of coding mistakes, and lack of granularity. ${ }^{30}$ For instance, NCDB codes for craniotomy, which could be performed to obtain biopsy rather than with the intent of resection. Consequently, we evaluated extent of resection in SEER to demonstrate the association between cytoreduction rather than craniotomy on survival. Our operative report review in IS also addresses this question, as surgeons intended to resect tumor in the vast majority of cases. Because many SEER data reporters also report to NCDB, we limited our SEER analysis to the question of cytoreduction and survival to avoid analyzing overlapping observations. The lack of information on 


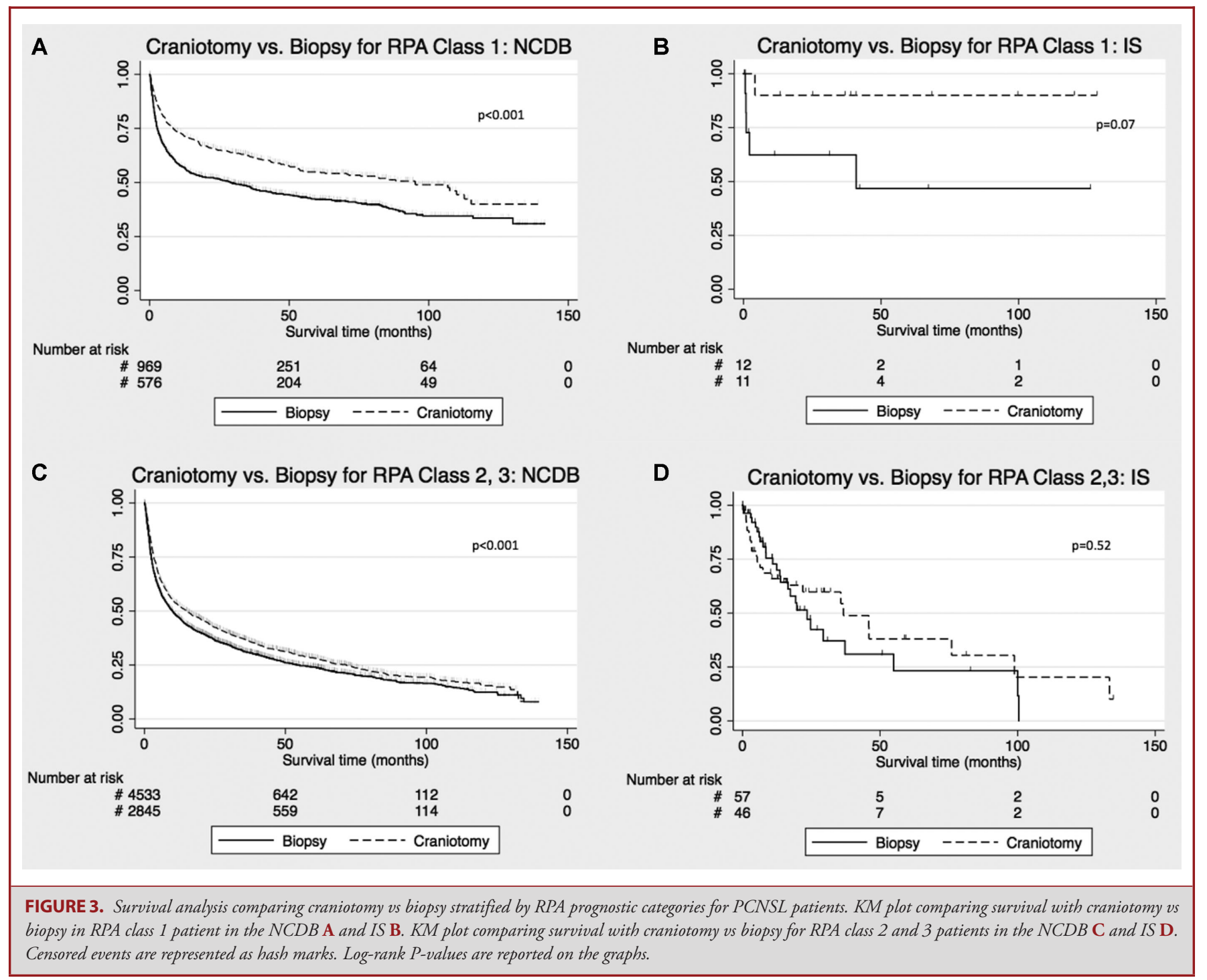

chemotherapy and radiotherapy in SEER also limited the utility of this database.

While 1 study reports that over $80 \%$ of patients have deep lesions ${ }^{50}$ another series found that $70 \%$ of patients with PCNSL presenting for neurosurgical evaluation have lobar lesions, ${ }^{23}$ and thus potentially resectable tumors. Our findings add to the growing body of evidence that using modern neurosurgical techniques, craniotomy for PCNSL is not only safe, ${ }^{32}$ but may also be associated with prolonged survival, particularly for those patients in favorable prognostic categories. However, given the methodological limitations of this and prior studies, current evidence falls short of demonstrating causality. Prospective studies to evaluate the management paradigm for PCNSL are warranted. Future studies should stratify patients based on prognostic factors, which likely influence survival after craniotomy.

\section{CONCLUSION}

Resection for PCNSL is considered high risk based on a series of smaller studies conducted prior to the implementation of modern neurosurgical techniques. The purpose of this study was to investigate the relationship between craniotomy and survival for PCNSL, building on recent reports re-evaluating the current paradigm. We used a retrospective analysis of over 9000 patients in 3 complementary datasets. We report an association between craniotomy and survival, most pronounced in subgroups with favorable prognostic factors. In contrast to prior literature, this study has a long follow-up time, large number of observations, and applicability to uncertain real-world practice. While methodological limitations preclude the demonstration of causality, this study adds to the evidence that prospective trials re-evaluating the 


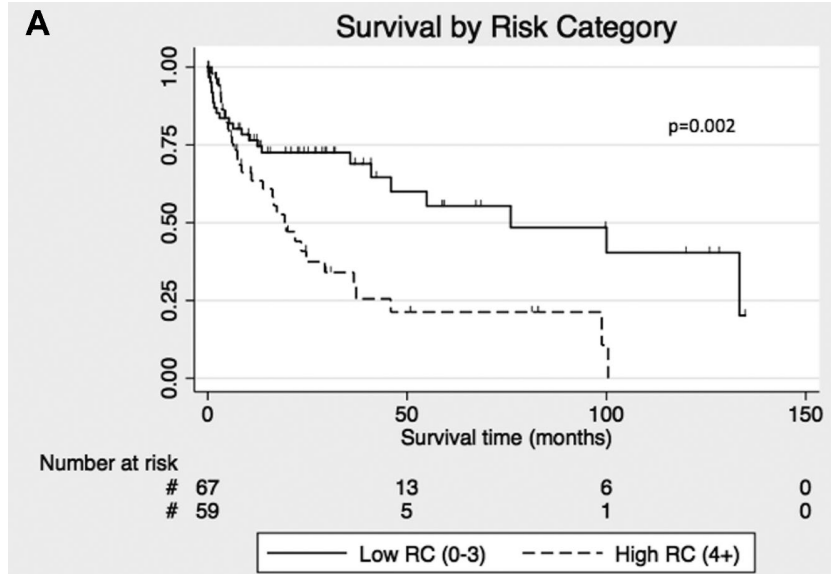

B

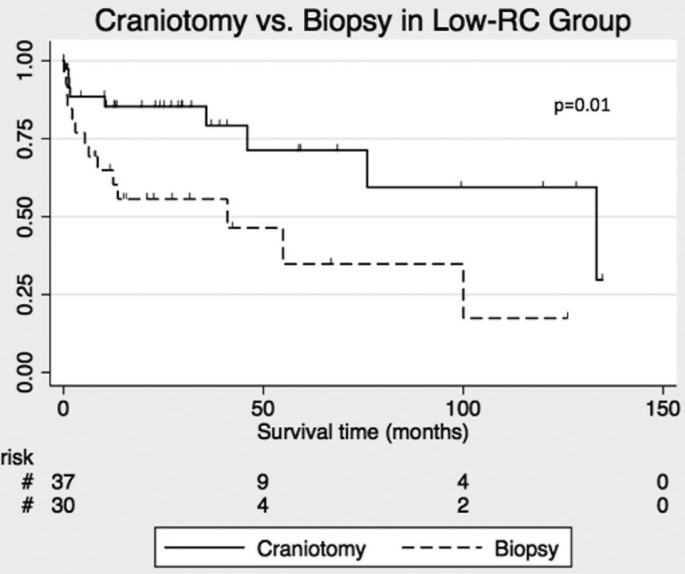

C

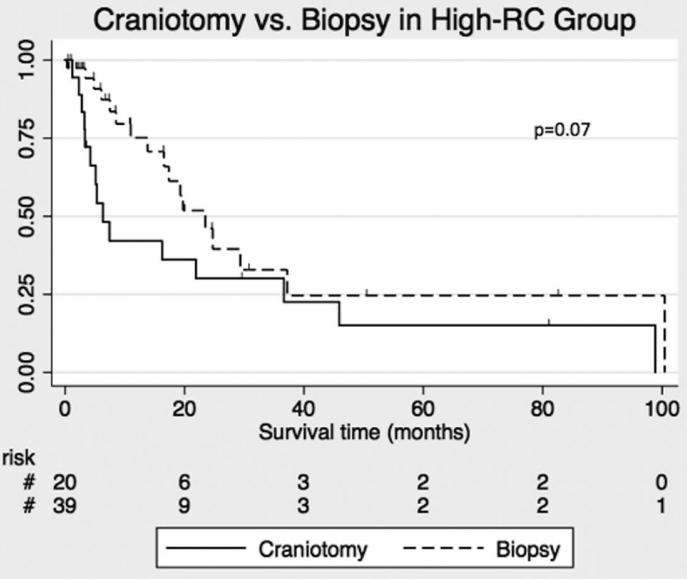

FIGURE 4. Risk category stratification analysis and its effects on survival differences between craniotomy and biopsy for PCNS patients in the institutional database. A, $R C$ stratification of low- $R C$ (score 0-3) vs high- $R C$ (score 4+) shows a significantly longer survival for low- $R C$ on the $K M$ analysis. B, KM plot comparing survival with craniotomy vs biopsy for low-RC patients with PCNSL, and in $\mathrm{C}$, high-RC patients. Censored events are represented as hash marks. Log-rank P-values are reported on the graphs.
TABLE 3. Clinical Risk Category Scale for Calculating Surgical Risk in Patients With PCNSL

\begin{tabular}{|ll|}
\hline \multicolumn{1}{|c|}{ Risk factor } & Point score \\
\hline Difficulty with activities of daily living & 1 \\
History of diabetes mellitus & 1 \\
Lung or respiratory disease & 1 \\
Congestive heart failure & 1 \\
History of myocardial infarction & 1 \\
Other cardiac disease & 1 \\
Arterial hypertension & 1 \\
Clouding, delirium, or cognitive impairment & 1 \\
History of Transient Ischemic Attack (TIA) & 1 \\
History of stroke & 1 \\
Peripheral vascular disease & 1 \\
Age $>$ 55 yr & 1 \\
Multiple CNS lesions & 1 \\
Deep lesion involving brainstem, basal ganglia, & 1 \\
corpus callosum, or periventricular area & \\
\hline
\end{tabular}

Total score of 4 or more indicates high surgical risk.

management of PCNSL are warranted, and provides a paradigm for guiding patient selection for such studies.

\section{Disclosures}

Funding was from the NIH office of the Director, Award Number 1DP5OD021356-01 (AMS); Diversity Recruitment Award by the Office of Senior Associate Provost for Faculty Diversity and Inclusion, Columbia University (AMS); and NIH Research Training in Aging Grant (T35 AG044303) (AM). The authors have no personal, financial, or institutional interest in any of the drugs, materials, or devices described in this article.

\section{REFERENCES}

1. Schabet M. Epidemiology of primary CNS lymphoma. J Neurooncol. 1999;43(3):199-201

2. Kasenda B, Ferreri AJ, Marturano E, et al. First-line treatment and outcome of elderly patients with primary central nervous system lymphoma (PCNSL)a systematic review and individual patient data meta-analysis. Ann Oncol. 2015;26(7):1305-1313.

3. Ferreri AJ, Reni $M$, Foppoli $M$, et al. High-dose cytarabine plus high-dose methotrexate versus high-dose methotrexate alone in patients with primary CNS lymphoma: a randomised phase 2 trial. Lancet North Am Ed. 2009;374(9700):1512-1520.

4. Batchelor T. Treatment and prognosis of primary central nervous system lymphoma. In April F. Eichler (Ed.) 2017. Retrieved July 18, 2016, from https://www.uptodate.com/contents/treatment-and-prognosis-of-primary-centralnervous-system-lymphoma.

5. Hoang-Xuan K, Bessell E, Bromberg J, et al. Diagnosis and treatment of primary CNS lymphoma in immunocompetent patients: guidelines from the European Association for Neuro-Oncology. Lancet Oncol. 2015;16(7):e322-e332.

6. Yun J, Iwamoto FM, Sonabend AM. Primary central nervous system lymphoma: a critical review of the role of surgery for resection. Arch Can Res. 2016;4(2):1-8.

7. Sanai N, Berger MS. Extent of resection influences outcomes for patients with gliomas. Rev Neurol (Paris). 2011;167(10):648-654.

8. Lacroix M, Abi-Said D, Fourney DR, et al. A multivariate analysis of 416 patients with glioblastoma multiforme: prognosis, extent of resection, and survival. J Neurosurg. 2001;95(2):190-198. 
9. Laws ER, Parney IF, Huang W, et al. Survival following surgery and prognostic factors for recently diagnosed malignant glioma: data from the Glioma Outcomes Project. J Neurosurg. 2003;99(3):467-473.

10. Brown PD, Maurer MJ, Rummans TA, et al. A prospective study of quality of life in adults with newly diagnosed high-grade gliomas: the impact of the extent of resection on quality of life and survival. Neurosurgery. 2005;57(3):495-504.

11. Jakola AS, Myrmel KS, Kloster R, et al. Comparison of a strategy favoring early surgical resection vs a strategy favoring watchful waiting in low-grade gliomas. JAMA. 2012;308(18):1881-1888.

12. Patchell RA, Tibbs PA, Walsh JW, et al. A randomized trial of surgery in the treatment of single metastases to the brain. N Engl J Med. 1990;322(8):494-500.

13. Henry JM, Heffner RR, Jr, Dillard SH, Earle KM, Davis RL. Primary malignant lymphomas of the central nervous system. Cancer. 1974;34(4):1293-1302.

14. Jellinger K, Radaskiewicz TH, Slowik F. Primary malignant lymphomas of the central nervous system in man. Acta Neuropathol Suppl. 1975;6(Suppl 6):95-102.

15. Berry MP, Simpson WJ. Radiation therapy in the management of primary malignant lymphoma of the brain. Int J Radiat Oncol Biol Phys. 1981;7(1): 55-59.

16. Pollack IF, Lunsford LD, Flickinger JC, Dameshek HL. Prognostic factors in the diagnosis and treatment of primary central nervous system lymphoma. Cancer. 1989;63(5):939-947.

17. Murray K, Kun L, Cox J. Primary malignant lymphoma of the central nervous system. J Neurosurg. 1986;65(5):600-607.

18. DeAngelis LM, Yahalom J, Heinemann MH, Cirrincione C, Thaler HT, Krol G. Primary CNS lymphoma: combined treatment with chemotherapy and radiotherapy. Neurology. 1990;40(1):80-80.

19. Hayakawa T, Takakura $\mathrm{K}$, Abe $\mathrm{H}$, et al. Primary central nervous system lymphoma in Japan. J Neurooncol. 1994;19(3):197-215.

20. Davies KG, Cole GC, Weeks RD. Twenty-year survival following excision of primary CNS lymphoma without radiation therapy: case report. Br J Neurosurg. 1994;8(4):487-491.

21. Tomlinson FH, Kurtin PJ, Suman VJ, et al. Primary intracerebral malignant lymphoma: a clinicopathological study of 89 patients. J Neurosurg. 1995;82(4):558-566.

22. Sonstein W, Tabaddor K, Llena JF. Solitary primary CNS lymphoma: long term survival following total resection. Med Oncol. 1998;15(1):61-65.

23. Bataille B, Delwail V, Menet E, et al. Primary intracerebral malignant lymphoma: report of 248 cases. J Neurosurg. 2000;92(2):261-266.

24. Caroli E, Acqui M, Ferrante L. Primary cerebral lymphoma: a retrospective study in 22 immunocompetent patients. Tumori. 2004;90(3):294-298.

25. Bellinzona M, Roser F, Ostertag H, Gaab RM, Saini M. Surgical removal of primary central nervous system lymphomas (PCNSL) presenting as space occupying lesions: a series of 33 cases. Eur J Surg Oncol. 2005;31(1):100-105.

26. Zebian B, Vergani F, Lavrador JP, et al. Recent technological advances in pediatric brain tumor surgery. CNS Oncol. 2017;6(1):71-82.

27. Neira JA, Ung TH, Sims JS, et al. Aggressive resection at the infiltrative margins of glioblastoma facilitated by intraoperative fluorescein guidance. $J$ Neurosurg. 2016;127(1):111-122

28. Stummer W, Pichlmeier U, Meinel T, et al. Fluorescence-guided surgery with 5-aminolevulinic acid for resection of malignant glioma: a randomised controlled multicentre phase III trial. Lancet Oncol. 2006;7(5):392-401.

29. Bilimoria KY, Stewart AK, Winchester DP, Ko CY. The National Cancer Data Base: a powerful initiative to improve cancer care in the United States. Ann Surg Oncol. 2008;15(3):683-690.

30. Davis FG, McCarthy BJ, Berger MS. Centralized databases available for describing primary brain tumor incidence, survival, and treatment: Central Brain Tumor Registry of the United States; Surveillance, Epidemiology, and End Results; and National Cancer Data Base. Neuro Oncol. 1999;1(3):205-211.

31. Abrey LE, Ben-Porat L, Panageas KS, et al. Primary central nervous system lymphoma: the Memorial Sloan-Kettering Cancer Center prognostic model. J Clin Oncol. 2006;24(36):5711-5715.

32. Cloney MB, Sonabend AM, Yun J, et al. The safety of resection for primary central nervous system lymphoma: a single institution retrospective analysis. J Neurooncol. 2017;132(1):189-197.

33. Ferreri AJ, Blay JY, Reni M, et al. Prognostic scoring system for primary CNS lymphomas: the International Extranodal Lymphoma Study Group experience. J Clin Oncol. 2003;21(2):266-272.

34. Clegg A, Young J, Iliffe S, Rikkert MO, Rockwood K. Frailty in elderly people. Lancet North Am Ed. 2013;381(9868):752-762.
35. Clegg A, Young J. The frailty syndrome. Clin Med. 2011;11(1):72-75.

36. Cloney M, D’Amico R, Lebovic J, et al. Frailty in geriatric glioblastoma patients: a predictor of operative morbidity and outcome. World Neurosurg. 2016;89:362367. doi: 10.1016/j.wneu.2015.12.096

37. Rockwood K, Howlett SE, MacKnight C, et al. Prevalence, attributes, and outcomes of fitness and frailty in community-dwelling older adults: report from the Canadian study of health and aging. J Gerontol A Biol Sci Med Sci. 2004;59(12):1310-1317.

38. Rockwood K, Song X, MacKnight C, et al. A global clinical measure of fitness and frailty in elderly people. Can Med Assoc J. 2005;173(5):489-495.

39. Karam J, Tsiouris A, Shepard A, Velanovich V, Rubinfeld I. Simplified frailty index to predict adverse outcomes and mortality in vascular surgery patients. Ann Vasc Surg. 2013;27(7):904-908.

40. Adams P, Ghanem T, Stachler R, Hall F, Velanovich V, Rubinfeld I. Frailty as a predictor of morbidity and mortality in inpatient head and neck surgery. JAMA Otolaryngol Head Neck Surg. 2013;139(8):783-789.

41. Tsiouris A, Hammoud ZT, Velanovich V, Hodari A, Borgi J, Rubinfeld I. A modified frailty index to assess morbidity and mortality after lobectomy. J Surg Res. 2013;183(1):40-46

42. Allison PD, Allison PD. Event History and Survival Analysis. 2nd edn. Los Angeles: Sage; 2014

43. Weller M, Martus P, Roth P, Thiel E, Korfel A, German PSG. Surgery for primary CNS lymphoma? Challenging a paradigm. Neuro Oncol. 2012;14(12):1481-1484.

44. Jelicic J, Todorovic Balint M, Raicevic S, et al. The possible benefit from total tumour resection in primary diffuse large B-cell lymphoma of central nervous system - a one-decade single-centre experience. Br J Neurosurg. 2016;30(1):80-85.

45. Lee HS, Park LC, Lee EM, et al. Comparison of therapeutic outcomes between surgical resection followed by R-CHOP and R-CHOP alone for localized primary intestinal diffuse large B-cell lymphoma. Am J Clin Oncol. 2014;37(2):182-187.

46. Attarbaschi A, Beishuizen A, Mann G, et al. Children and adolescents with follicular lymphoma have an excellent prognosis with either limited chemotherapy or with a "Watch and wait" strategy after complete resection. Ann Hematol. 2013;92(11):1537-1541.

47. Lin CH, Kuo KT, Chuang SS, et al. Comparison of the expression and prognostic significance of differentiation markers between diffuse large B-cell lymphoma of central nervous system origin and peripheral nodal origin. Clin Cancer Res. 2006;12(4):1152-1156.

48. Rubenstein JL, Shen A, Batchelor TT, Kadoch C, Treseler P, Shuman MA. Differential gene expression in central nervous system lymphoma. Blood. 2009;113(1):266-267.

49. Sonabend AM, Zacharia BE, Cloney $\mathrm{MB}$, et al. Defining glioblastoma resectability through the wisdom of the crowd. Neurosurgery. 2017;80(4):590-601.

50. Ferreri AJ, Cwynarski K, Pulczynski E, et al. Chemoimmunotherapy with methotrexate, cytarabine, thiotepa, and rituximab (MATRix regimen) in patients with primary CNS lymphoma: results of the first randomisation of the International Extranodal Lymphoma Study Group-32 (IELSG32) phase 2 trial. Lancet Haematol. 2016;3(5):e217-e227.

Supplemental digital content is available for this article at www neurosurgery-online.com.

Supplemental Digital Content 1. Figure. T1 post-contrast MRI of a biopsied deep PCNSL lesion that underwent a biopsy (left) and a superficial lesion that was managed with a craniotomy for resection (right).

Supplemental Digital Content 2. Figure. Further analysis of proportional hazards assumption violation in the multivariable Cox model in NCDB. A, Log$\log$ plot, and B, K-M vs predicted curves of craniotomy vs biopsy landmarked at 30 d. C, Log-log plot, and D, K-M vs predicted curves of chemotherapy vs no chemotherapy. E Log-log plots, and F, K-M vs predicted curves of radiation vs no radiation treatment.

Supplemental Digital Content 3. Figure. RPA stratification distinguishes prognostic categories for PCNSL patients. A, KM plot for RPA1 class 1 vs higher on NCDB-PUF. B, KM plot for RPA1 class 1, class 2 and class 3 in the institutional dataset. Censored events are represented as hash marks. Log-rank $P$-values are reported on the graphs. 


\section{COMMENTS}

$\mathbf{T}$ he authors used 3 datasets (NCDB, SEER, institutional series) to retrospectively examine the relationship between craniotomy and survival in PCNSL. They appropriately acknowledge the limitations of their study and discuss some of the obvious pitfalls, including: 1) the fact that more resectable lesions may confer a better prognosis, 2) patients selected for craniotomy may be better surgical candidates and thus could be expected to have longer survival, and 3) some "craniotomies" for PCNSL are actually "glorified biopsies" without intent of aggressive resection. The authors attempted to address some of these issues by utilizing a risk category classification to stratify patients into similar groups and by evaluating extent of resection in the SEER and institutional series. Given that the current paradigm against cytoreductive surgery for PCNSL is based on older studies, and in light of multiple threads of data, including this study, suggesting a positive relationship between extent of resection and survival, I agree with the authors' call for future prospective studies to evaluate resection of lobar PCNSL lesions utilizing modern neurosurgical techniques.

\section{Ramsey Ashour} Austin, Texas of a cerebral lymphoma mass. Their overall findings, that resection of a lymphoma mass appears to impart survival benefit should be interpreted carefully. The selection biases inherent in choosing resective candidates for surgery in undiagnosed lesions will naturally favor those with single, more superficial lesions in patients with more favorable survival characteristics. The data does not support the practice of chasing diffuse lymphoma lesions.

Nonetheless, given the strength of the data, analyzed carefully by the authors, I think it is reasonable to conclude that if a surgeon has performed a craniotomy for an undiagnosed mass lesion in non-eloquent brain, and the intraoperative pathology suggests lymphoma, it is proper to resect the remaining mass to the extent the surgeon considers safe rather than halting the procedure. Finishing the resection has been common practice-and common sense-for many. This publication gives strong rationale for this action, as long as it can be accomplished without significant morbidity.

The benefits of resection are maximal at early follow-up and fade with longer-term follow-up, as is the case with most malignant disease. However, in their institutional database analysis, the authors identified a low-risk group via recursive partition analysis that maintains the benefits of resection over long-term follow-up. A prospective study would help to quantify this benefit.
$\mathbf{U}$ sing 3 retrospective databases (NCDB, SEER, and an institutional database) the authors have put together an important and thoughtful analysis of the questions surrounding the value of resection
Richard W. Byrne Chicago, Illinois 